\title{
A new implication for strong interactions if large, direct CP violation in $\bar{B}^{0}\left(B^{0}\right) \rightarrow \pi^{+} \pi^{-}$is confirmed
}

\author{
Saul Barshay and Georg Kreyerhoff \\ III. Physikalisches Institut \\ RWTH Aachen \\ D-52056 Aachen \\ Germany
}

January 11, 2014

\begin{abstract}
We show that the large, direct CP-violation parameter $A_{\pi \pi}=-C_{\pi \pi}$, reported by the BELLE collaboration in the decays $\bar{B}^{0}\left(B^{0}\right) \rightarrow \pi^{+} \pi^{-}$, implies an unusual situation in which the presence of a very large difference between two strong-interaction phases $\left(\sim-110^{\circ}\right)$ plays an essential role. We make the demonstration within a model of strong, two-body quasi-elastic interactions between physical hadrons. The model can accommodate a large difference between two strong-interaction phases, for which it provides a natural enhancement.
\end{abstract}

Two experiments have very recently published results concerning $\mathrm{CP}$ violation in the decays $\bar{B}^{0}\left(B^{0}\right) \rightarrow \pi^{+} \pi^{-}[1,2,3]$. The results involve measurements of the two parameters which determine mixing-induced and direct $\mathrm{CP}$-violation. Direct CP-violation would occur even if the sizable mixing[4] between $B^{0}$ and $\bar{B}^{0}$ caused by the known [4] mass difference $\Delta m_{d}$ between the states $\left(p B^{0} \mp q \bar{B}^{0}\right) / \sqrt{2}$, were to vanish (i. e. $\Delta m_{d} \rightarrow 0$ ). When one recalls that more than 25 years passed after the discovery of $\mathrm{CP}$ violation[5], before direct $\mathrm{CP}$ violation in the $K^{0}-\bar{K}^{0}$ system was established by different experiments $[6,7,8]$, it is clear that the significance of the present single experimental indication $[1,3]$ for large, direct $\mathrm{CP}$ violation in the $B^{0}-$ $\bar{B}^{0}$ system warrants careful consideration. The two general empirical parameters mentioned above are, of course, related through the parameters of the standard model and certain dynamical assumptions [1, 2]. However in the decay mode in which CP violation in the $B^{0}-\bar{B}^{0}$ system was discovered $[9,10]$, that due to mixing is large $[9,10]$, whereas direct $\mathrm{CP}$ violation is not detected [9]. It is a general requirement $[11,12]$ that significant elastic and/or inelasticity-induced, strong-interaction phases (at least two different phases) be present, in order for direct $\mathrm{CP}$ violation to be manifest in the decay amplitudes for particle and antiparticle. ${ }^{F 1}$ In this paper we demonstrate within an explicit and clear dynamical model involving physical hadrons, that if the large, direct $\mathrm{CP}$ violation recently reported $[1,3]$ is confirmed, then a most unusual situation must hold for the two necessary strong-interaction phases. This is our main purpose. The model can accommodate, in principle, the

\footnotetext{
${ }^{F 1}$ In 11 is one of the first published calculations which explicitly demonstrates the necessary role of different inelasticity-induced, as well as elastic, strong-interaction phases among physical hadrons in CP violation (sometimes referred to as "soft" final-state interactions, in the ensuing popular literature).
} 
necessary generation of a large difference between two strong-interaction phases of about $-110^{\circ}$. It provides a natural enhancement for the phase difference.

The time evolution of the decays $\bar{B}^{0}\left(B^{0}\right) \rightarrow \pi^{+} \pi^{-}$is described $[1,2]$ by ${ }^{F 2}$

$$
f_{ \pm}(\Delta t)=\frac{e^{-|\Delta t| / \tau_{B^{0}}}}{4 \tau_{B^{0}}}\left\{1 \pm S_{\pi \pi} \sin \left(\Delta m_{d} \Delta t\right) \mp C_{\pi \pi} \cos \left(\Delta m_{d} \Delta t\right)\right\}
$$

where the upper (lower) sign refers to $\overline{B^{0}}\left(B^{0}\right)$ decay (with a $B^{0}\left(\bar{B}^{0}\right)$ tagged). The phenomenological parameters[13] $S_{\pi \pi}$ and $C_{\pi \pi}$, for mixing-induced and direct CP violation respectively, are defined by

$$
S_{\pi \pi}=\frac{2 \operatorname{Im} \lambda}{1+|\lambda|^{2}}, \quad C_{\pi \pi}=\frac{1-|\lambda|^{2}}{1+|\lambda|^{2}}
$$

with $\lambda=(q / p)\left(\bar{B}_{\pi \pi} / B_{\pi \pi}\right)$. The states (heavy (H) and light (L)) with mass difference $\Delta m_{d}[13]$ are

$$
\left|B_{H, L}>=\left(p\left|B^{0}>\mp q\right| \bar{B}^{0}>\right) / \sqrt{2} ; \quad\right| q / p \mid \cong 1
$$

Here $\bar{B}_{\pi \pi}$ and $B_{\pi \pi}$ denote the complex decay amplitudes for $\bar{B}^{0}\left(B^{0}\right) \rightarrow \pi^{+} \pi^{-}$, respectively. In the model developed below, these amplitudes depend upon two strong-interaction phases, and upon a CP-violating weak-interaction phase; an explicit dependence is exhibited in Eqs. $(7,8)$ below. The BELLE[1,3] and BABAR[2] results are:

$$
\begin{array}{cl}
\text { BELLE : } & S_{\pi \pi}=-1.23 \pm 0.41(\text { stat }){ }_{-0.07}^{+0.08}(\text { syst }) \\
& C_{\pi \pi}=-A_{\pi \pi}=-0.77 \mp 0.27 \text { (stat) } \mp 0.08 \text { (syst) } \\
\text { BABAR : } & S_{\pi \pi}=+0.02 \pm 0.34(\text { stat }) \pm 0.05 \text { (syst) } \\
& C_{\pi \pi}=-A_{\pi \pi}=-0.30 \pm 0.25(\text { stat }) \pm 0.04 \text { (syst) }
\end{array}
$$

As we shall see below, a remarkable feature of the BELLE data is the large magnitude of $C_{\pi \pi}\left(-A_{\pi \pi}\right),{ }^{F 3}$ the parameter which describes direct $\mathrm{CP}$ violation (i. e. is present for $\Delta m_{d} \rightarrow 0$ ).

We begin the analysis with the determination of two strong-interaction eigenstates ${ }^{F 4}$ which mix the final $\pi^{+} \pi^{-}$system (the component with isospin zero) at a total (c. m. ) energy of $m_{B^{0}}=5.28 \mathrm{GeV}$, with another two-particle system of distinctly different particle content, namely $\eta \eta_{c} \cdot[12]{ }^{F 5}$ This particular system is mixed by quasi-elastic strong interaction $[11,12]$ with $\pi \pi \cdot{ }^{F 5}$ The reaction is quasielastic at a c. m. energy of $5.28 \mathrm{GeV}$, the momentum in the $\eta \eta_{c}$ system is 0.7 times that in the $\pi \pi$ system. The $c \bar{c}$ component which is implicit in the constitution of the system ${ }^{F 5} \eta \eta_{c}$, brings the complex phase $\delta$ ( or $\left.\delta_{13}\right)[14]$ of the CKM matrix

\footnotetext{
${ }^{F 2}$ We use the notation of [2]. One must note that the parameter for direct $\mathrm{CP}$ violation $C_{\pi \pi}$, is the same as $(-1)$ times the parameter denoted by $A_{\pi \pi}$ in $[1,3]$.

${ }^{F 3}$ The physical boundary is at $\left(S_{\pi \pi}^{2}+C_{\pi \pi}^{2}\right)=1$. Approximate formulae from our model and from other models [3], may allow larger values near to the boundary, for certain values of the parameters. So does the present BELLE data, presumably from a statistical fluctuation. $[1,3]$

${ }^{F 4}$ The consideration of two eigenstates is a minimal idealization which allows one to see clearly the essential features of the strong and weak-interaction elements in the physics, when the $\eta \eta_{c}(c \bar{c})$ particle (quark) content of the mixed system is considered. All three quark generations come into play, through charged currents.

${ }^{F 5}$ One could equally well consider the system $\eta^{\prime} \eta_{c}$. Both systems were used in [12], where stronginteraction phases were calculated which are relevant for estimating sizable direct CP-violating asymmetries in the charged decay modes $B^{ \pm} \rightarrow\left(\pi^{ \pm} \eta, \pi^{ \pm} \eta^{\prime}, \pi^{ \pm} \eta_{c}\right)$. A CP-violating asymmetry in a charged-particle decay mode remains to be discovered (for $B$ or $K$ ). BELLE may be on the track (private communication from K. Abe).
} 
(necessary for $\mathrm{CP}$ violation in the standard-model weak interaction) directly into play in the decay amplitudes $\bar{B}_{\pi \pi}$ and $B_{\pi \pi}$ (Eq. (6) below). Consider a two-by-two strong-interaction $K$ matrix for quasi-elastic scattering of the form

$$
\left.K=\pi_{\eta \pi} \quad \begin{array}{cc}
\pi \pi & \eta \eta_{c} \\
0 & -2 \sqrt{p_{\pi} p_{\eta_{c}}} \\
-2 \sqrt{p_{\pi} p_{\eta_{c}}} & 0
\end{array}\right) \times e / \sqrt{2}\left(4 \pi m_{B^{0}}\right)
$$

The momenta in the two systems are respectively, $p_{\pi} \cong\left(m_{B^{0}} / 2\right), p_{\eta_{c}} \cong 0.7\left(m_{B^{0}} / 2\right)$. The single dimensionless parameter $e$ controls the strength of the effective "scattering length", $(\sqrt{2} e) /\left(4 \pi m_{B^{0}}\right),{ }^{F 6}$ which is taken as $>0$. A priori, a parameter like $e$ is of order unity.[12] We shall allow $e$ to be "enhanced", in order to see what is necessary to represent the BELLE data. We do not consider an effective "scattering length" larger than a few times $\left(1 / m_{B^{0}}\right)$, that is $<(1 / \mathrm{GeV})$, corresponding to a maximal strong-interaction phase of about $60^{\circ}$ in magnitude. Unitarity is respected by using $1 /(1+i K)$ in the decay amplitudes[12], leading to the Watson strong-interaction phase factors $e^{i \Delta_{1,2}}$ for the eigenstates. The eigenstates of $K$ are simply $\mid 1>=\left(\left|\pi^{+} \pi^{-}>+\right| \eta \eta_{c}>\right) / \sqrt{2}$ with eigenvalue phase $\Delta_{1}$ given by $\tan \Delta_{1}=+\left(\sqrt{2 p_{\pi} p_{\eta_{c}}} e\right) /\left(4 \pi m_{B^{0}}\right)$, and $\mid 2>=\left(\left|\pi^{+} \pi^{-}>-\right| \eta \eta_{c}>\right) / \sqrt{2}$ with eigenvalue phase $\Delta_{2}$ given by $\tan \Delta_{2}=-\left(\sqrt{2 p_{\pi} p_{\eta_{c}}} e\right) /\left(4 \pi m_{B^{0}}\right)$. Note that there are the two essential strong-interaction phases, and that these are opposite in sign. This feature leads to an immediate doubling of the magnitude of the phase difference $\left(\Delta_{2}-\Delta_{1}\right)$, and the direct CP-violating asymmetry parameter $C_{\pi \pi}$ is proportional to $\sin \left(\Delta_{2}-\Delta_{1}\right)$. This doubling is an important effect obtained in this model, also because it can take individual phases in the fourth and first quadrants into a phase difference in the third quadrant, with the resulting negative sign for the cosine and sine of the phase difference. It is the negative cosine which gives rise to an enhancement of the magnitude of $C_{\pi \pi}$, in Eq. (10) below. The state $\mid \pi^{+} \pi^{-}>=(|1>+| 2>) / \sqrt{2}$; the explicit dependence on the strong-interaction phases in the decay amplitudes can be exhibited as

$$
\begin{aligned}
\bar{B}_{\pi \pi} & =e^{i \Delta_{1}}\left(A_{1}+A_{2} e^{i\left(\Delta_{2}-\Delta_{1}\right)}\right) \\
B_{\pi \pi} & =e^{i \Delta_{1}}\left(A_{1}^{*}+A_{2}^{*} e^{i\left(\Delta_{2}-\Delta_{1}\right)}\right)
\end{aligned}
$$

The two complex amplitudes $A_{1,2}$ involve the weak interactions; explicitly the KM phase $\delta$ from the quark mixing matrix. Within this model with eigenstates $\left(\left|\pi^{+} \pi^{-}> \pm\right| \eta \eta_{c}>\right) / \sqrt{2}$, we are led to an ansatz for the explicit forms $A_{1,2}$,

$$
\begin{aligned}
A_{1}= & A e^{-i \delta}\left(1+a e^{i \delta}\right) \\
A_{2}= & A e^{-i \delta}\left(1-a e^{i \delta}\right) \\
& A \text { real }
\end{aligned}
$$

with $a \cong\left(s_{2} / s_{3}\right) f_{c \bar{c}}=\tilde{s} f_{c \bar{c}} \sim \tilde{s}$. Here $f_{c \bar{c}}$ represents a "fraction" for a $c \bar{c}$ component in the system $\eta \eta_{c}$; we use $f_{c \bar{c}} \sim 1$ in numerical estimates. The ratio of the sines of small mixing angles $\left(s_{2} / s_{3}\right)=\tilde{s}>1$ (we use $\tilde{s} \sim 2.5$ in numerical estimates[14]), appears as the result of the relative values of different elements of the CKM matrix (in its original form[15], or as $\left(s_{12} s_{23} / s_{13}\right)$ in the "standard" form[14]). The relevant elements involve that for $b \rightarrow c$ times that leading to $\bar{c} d$, relative to that for $b \rightarrow u$ times that leading to $\bar{u} d$. (We approximate the cosines of the small CKM angles as

\footnotetext{
${ }^{F 6}$ For simplicity, we do not deal with diagonal elements. For elastic scattering, it is the diagonal elements which are non-zero, and these $K$ matrix elements are simply the negative of the tangent of the elastic phase shifts. Thus, here the quantity $\left(\sqrt{2} e /\left(4 \pi m_{B^{0}}\right)\right.$ is the magnitude of an effective "scattering length" which acts as a measure of the strength of the two-body quasi-elastic interaction $\pi^{+} \pi^{-} \rightarrow \eta \eta_{c}$, at c. m. energy of $m_{B^{0}}=5.28 \mathrm{GeV}$.
} 
$\sim 1$.) Thus, the amplitudes $A_{1,2}$ involve two different CP-violating phases $\delta_{1,2}$, as functions of $\delta$.

$$
\begin{aligned}
A_{1} & =A N_{1} e^{-i \delta} e^{i \delta_{1}} ; \tan \delta_{1}=\frac{\tan \delta}{(1+(1 / \tilde{s} \cos \delta))} \\
A_{2} & =A N_{2} e^{-i \delta} e^{i \delta_{2}} ; \tan \delta_{2}=\frac{\tan \delta}{(1-(1 / \tilde{s} \cos \delta))} \\
\text { with } N_{1,2} & =\left\{(1 \pm \tilde{s} \cos \delta)^{2}+(\tilde{s} \sin \delta)^{2}\right\}^{1 / 2}
\end{aligned}
$$

The phase difference $\left(\delta_{2}-\delta_{1}\right)$ is not zero unless $\delta \rightarrow 0$ (i. e. $\delta_{1}=\delta_{2}=0$ ). Using Eqs. $(6,7)$ we have, in the model

$$
\begin{aligned}
\bar{B}_{\pi \pi} & =A^{\prime} e^{-i \delta} e^{i\left(\Delta_{1}+\delta_{1}\right)}\left\{r+e^{i\left(\Delta_{2}-\Delta_{1}\right)} e^{i\left(\delta_{2}-\delta_{1}\right)}\right\} \\
B_{\pi \pi} & =A^{\prime} e^{+i \delta} e^{i\left(\Delta_{1}-\delta_{1}\right)}\left\{r+e^{i\left(\Delta_{2}-\Delta_{1}\right)} e^{-i\left(\delta_{2}-\delta_{1}\right)}\right\} \\
\text { with } r & =\left(N_{1} / N_{2}\right), \quad A^{\prime}=A N_{2}
\end{aligned}
$$

Then

$$
\begin{aligned}
\lambda & =\left(\frac{q}{p}\right)\left(\frac{\bar{B}_{\pi \pi}}{B_{\pi \pi}}\right) \\
& =e^{-i(2 \beta+2 \delta)} e^{i 2 \delta_{1}}\left\{\frac{r+e^{i\left(\Delta_{2}-\Delta_{1}\right)} e^{i\left(\delta_{2}-\delta_{1}\right)}}{r+e^{i\left(\Delta_{2}-\Delta_{1}\right)} e^{-i\left(\delta_{2}-\delta_{1}\right)}}\right\}
\end{aligned}
$$

using $[13,14](q / p)=e^{-i 2 \beta}, 2 \beta \sim 45^{\circ}$. We thus obtain explicit formulae for the $C_{\pi \pi}$ and $S_{\pi \pi}$ in Eq. (2a), using the model; we use[14] $\delta \sim 45^{\circ}$ in numerical estimates.

$$
\begin{aligned}
C_{\pi \pi}= & +\frac{\left(\sin \Delta_{21}\right)\left(\sin \delta_{21}\right)}{\left(\frac{1+r^{2}}{2 r}\right)+\left(\cos \Delta_{21}\right)\left(\cos \delta_{21}\right)} \\
S_{\pi \pi}= & +\cos \left(2 \beta+2 \delta-2 \delta_{1}\right)\left\{\frac{\left(r \cos \Delta_{21}+\cos \delta_{21}\right) \sin \delta_{21}}{\left(\frac{1+r^{2}}{2}\right)+r\left(\cos \Delta_{21}\right)\left(\cos \delta_{21}\right)}\right\} \\
& -\sin \left(2 \beta+2 \delta-2 \delta_{1}\right)
\end{aligned}
$$

with $r^{2} \cong 2.95 ; \tan \delta_{1} \cong 1 /(1+1 /(2.5 \times .7)) \cong 0.635 \Rightarrow \delta_{1} \cong 32.5^{\circ}$;

$\tan \delta_{2} \cong 1 /(1-1 /(2.5 \times .7)) \cong 2.32 \Rightarrow \delta_{2} \cong 66.5^{\circ}$ and thus $\delta_{21}=\left(\delta_{2}-\delta_{1}\right) \cong 34^{\circ}$; $\Delta_{21}=\left(\Delta_{2}-\Delta_{1}\right)$.

Clearly, as $\delta \rightarrow 0$ we have $\delta_{1,2} \rightarrow 0$ and $|\lambda| \rightarrow 1$; then $C_{\pi \pi} \rightarrow 0$ and $S_{\pi \pi} \rightarrow$ $-\sin 2 \beta \sim-0.7$, as measured in $[9,10]$. As stated after Eq. (3), the BELLE value for $A_{\pi \pi}=-C_{\pi \pi}$ is large; in the context of the above formula this requires a very large $\Delta_{21}$ to obtain an enhancement factor from the denominator. We consider $\Delta_{21} \cong-110^{\circ}$. As stated following Eq. (4), this is nearly the largest phase difference that we can consider within the framework of this model. Then from Eqs. (10), we calculate

$$
\begin{aligned}
C_{\pi \pi} & \sim-\frac{\left(\sin 110^{\circ}\right)\left(\sin 34^{\circ}\right)}{1.15+\left(\cos 110^{\circ}\right)\left(\cos 34^{\circ}\right)} \sim-0.6 \\
S_{\pi \pi} & \sim\left(\cos 70^{\circ}\right)\left\{\frac{\left(1.72 \cos 110^{\circ}+\cos 34^{\circ}\right)\left(\sin 34^{\circ}\right)}{1.97+1.72 \cos 110^{\circ} \cos 34^{\circ}}\right\}-\sin 70^{\circ} \\
& \sim-0.9
\end{aligned}
$$

Such a large difference between two strong-interaction phases is a surprising new 
result. ${ }^{F 7}$ From our discussion following Eq. (4),

$$
\begin{aligned}
& \Delta_{21}=\left\{\tan ^{-1}(-\omega)-\tan ^{-1} \omega\right\} \cong-110^{\circ} \\
& \Rightarrow \omega=\sqrt{p_{\pi} p_{\eta_{c}}}\left(\frac{\sqrt{2} e}{4 \pi m_{B_{0}}}\right)=\sqrt{p_{\pi} p_{\eta_{c}}} \mathcal{A} \sim 1.43
\end{aligned}
$$

and thus there is an effective "scattering length" of

$$
\mathcal{A}=\left(\frac{\sqrt{2} e}{4 \pi m_{B^{0}}}\right) \sim \frac{1.43}{\sqrt{p_{\pi} p_{\eta_{c}}}} \sim \frac{3.4}{m_{B^{0}}} \cong 0.65 / \mathrm{GeV}<1 / \mathrm{GeV}
$$

This dynamical model thus allows, in principle, for such a strong quasi-elastic interaction. ${ }^{F 8}$ However, in the context of two-body interactions of physical hadrons at $5.28 \mathrm{GeV}$ c. m. energy, the $\mathcal{A}$ in Eq. (13) appears rather large. For example, taking[12] $e \sim 1$ gives only a small $\mathcal{A} \sim 0.11 / m_{B^{0}} \cong 0.02 / \mathrm{GeV}$. Then, $\Delta_{21}=\left(\Delta_{2}-\Delta_{1}\right) \sim-2 \omega \sim-0.09$, which results in a much smaller parameter for direct $\mathrm{CP}$ violation

$$
C_{\pi \pi} \sim-0.025
$$

If there is a large $C_{\pi \pi}$, a measurable prediction for the decay mode $\bar{B}^{0}\left(B^{0}\right) \rightarrow$ $\eta \eta_{c}$ is that $\left(C_{\eta \eta_{c}}\right)_{\text {b.r. }}=-\left(C_{\pi \pi}\right)_{\text {b.r. }}$, where b.r. denotes multiplication of $C$ by the corresponding branching ratio. Also, from only the quasi-elastic strong-interactions which we have discussed here, the parameter for direct $\mathrm{CP}$ violation in $\bar{B}^{0}\left(B^{0}\right) \rightarrow$ $\pi^{0} \pi^{0}$ is $\left(C_{\pi \pi}^{0}\right)_{\text {b.r. }}=\left(C_{\pi \pi}\right)_{\text {b.r. }}$.

The main result of this paper is the sizable value calculated for $A_{\pi \pi}=-C_{\pi \pi}$. In connection with the present experimental results $[2,3]$ for $S_{\pi \pi}$, we note that there is a different simple possibility for the $S_{\pi \pi}$ calculated within the framework of Eqs. (10) obtained from this dynamical model. This involves the possibility within the model of $\delta_{1,2} \rightarrow \delta_{2,1}$ with $N_{1,2} \rightarrow N_{2,1}$, and $\Delta_{1,2} \rightarrow \Delta_{2,1}$. This illustrates the role of the first term for $S_{\pi \pi}$ in Eq. (11) which then gives rise to a somewhat smaller $-S_{\pi \pi} . C_{\pi \pi}$ is unchanged. This suggests that it is not natural to have a sizable $C_{\pi \pi}$ together with a very small $S_{\pi \pi}$. On the other hand, if final-state interactions are completely neglegible, $C_{\pi \pi} \rightarrow 0$; then we have $S_{\pi \pi} \rightarrow \sin (2 \beta+2 \delta)$, which could be $\sim 0$ if $(2 \beta+2 \delta)$ is $\sim 180^{\circ}$.

In summary, we have shown that the large, direct $\mathrm{CP}$-violating parameter $\left|C_{\pi \pi}\right|$ reported $[1,3]$ by the BELLE collaboration implies the presence of a very large difference between (at least) two strong-interaction phases. We have shown how strong, two-body quasi-elastic interactions between physical hadrons at $m_{B^{0}}=5.28$ $\mathrm{GeV}$, can accommodate such an unusual strong-interaction phase difference. ${ }^{F 8}$ This type of model might allow new possibilities for estimating the strong-interaction phases which are necessary for observable direct $\mathrm{CP}$ violation, in other two-body final states from $\bar{B}^{0}\left(B^{0}\right)$ decay.

S. B. thanks Lahlit Sehgal and Kazuo Abe for information.

\footnotetext{
${ }^{F 7}$ It is interesting that the BELLE collaboration has reached a similar conclusion about the necessity for a very large strong-interaction phase difference (also $\sim-110^{\circ}$, note Fig. 9 in [3]), using different phenomenological formulae which originate in quark dynamics, (but are similar in form to our Eq. (10)). However, there is no indication of how such a large phase difference might arise from high-energy strong interactions involving perturbative gluons and elementary quarks. Neither is it apparent how hypothetically different, strong-interaction phases for different amplitudes at this level, are carried over to the state of physical hadrons.

${ }^{F 8}$ Extension of the idea of using coupled channels ${ }^{F 5}$, to include $\eta^{\prime}, \eta(1295), \eta(1440)$, could increase the effective strength (as then roughly summarized by the parameter $\mathcal{A}$ in Eq. (13)).
} 


\section{Added note}

The BABAR collaboration has just reported the observation of the decays $B^{\mp} \rightarrow$ $\pi^{\mp} \eta$, and an interesting negative, direct CP-violating asymmetry (in hep-ex/0303039). In [12], minimal estimates of the strong-interaction phases are used in estimating the CP-violating asymmetries. Values of the $K$-matrix elements for the final-state strong interactions are taken, which are minimal in magnitude. These are likely to be larger, by a factor of at least 2 . The calculated asymmetries are multiplied by

this factor. S. B. thanks Janice Button-Schafer for helpful communications about the new BABAR results.

\section{References}

[1] BELLE Collab., K. Abe et al., Phys. Rev. Lett.89 (2002) 071801

[2] BABAR Collab., B. Aubert et al., Phys. Rev. Lett 89 (2002) 281802

[3] BELLE Collab., K. Abe et al., hep-ex/0301032, Feb. 2003

[4] ARGUS Collab., H. Albrecht et al., Phys. Lett. B192 (1987) 245

[5] J. H. Christenson, J. W. Cronin, V. L. Fitch and R. Turlay, Phys. Rev. Lett. 13 (1964) 138

[6] NA 31 Collab., H. Burkhardt et al., Phys. Lett. B206 (1988) 168

[7] N48 Collab., V. Fanti et al., Phys. Lett. B465 (1999) 335

[8] KTeV Collab., A. Alavi-Harati et al., Phys. Rev. Lett. 83 (1999) 22

[9] BABAR Collab., B. Aubert et al., Phys. Rev. Lett. 89 (2001) 091801

BABAR Collab., B. Aubert et al., hep-ex/0203007

[10] BELLE Collab., K. Abe et al., Phys. Rev. Lett. 87 (2001) 091802

BELLE Collab., K. Abe et al., hep-ex/0202027v2

[11] S. Barshay and J. Geris, Phys. Lett. B84 (1979) 319

[12] S. Barshay, D. Rein and L. Sehgal, Phys. Lett. B259 (1991) 475

[13] Y. Nir and H. R. Quigg, Ann. Rev. Nucl. Part. Sci. 42 (1992) 211

[14] Particle Physics Booklet, July 2002, pages 165-172

Particle Data Group, K. Hagiwara et al., Phys. Rev. D66 (2002) 010001-1

[15] M. Kobayashi and J. Maskawa, Prog. Theor. Phys. 49 (1973) 652 